\title{
A Modified Method for Order Reduction of Large Scale Discrete Systems
}

\author{
Dr. G.Saraswathi \\ Dept. of EEE, GIT, GITAM University \\ Visakhapatanam, India
}

\begin{abstract}
In this paper, an effective procedure to determine the reduced order model of higher order linear time invariant discrete systems is discussed. A new procedure has been proposed for evaluating Time moments of the original high order system. Numerator and denominator polynomials of reduced order model are obtained by considering first few redefined time moments of the original high order system. The proposed method has been verified using numerical examples.
\end{abstract}

Keywords- order reduction; eigen values; large scale discrete systems; modeling.

\section{INTRODUCTION}

The rapid advancements in science and technology led to extreme research in large scale systems. As a result the overall mathematical complexity increases. The computational procedure becomes difficult with increase in dimension. Therefore high-order models are difficult to use for simulation, analysis or controller synthesis. So it is not only desirable but necessary to obtain satisfactory reduced order representation of such higher order models. Here the objective of the model reduction of high order complex systems is to obtain a Reduced Order Model(ROM) that retains and reflects the important characteristics of the original system as closely as possible.

Several methods are available in the literature for largescale system modeling. Most of the methods based on the original continued fraction expansion technique $[1,2]$ fail to retain the stability of the original systems in the reduced order models. To overcome this major drawback, alternative methods have been suggested but the common limitation of such extension is that in some cases, they may generate models of order even higher than that of the original system [1-3].

Modal-Padé methods [4] use the concept of the dominant poles and matching the few initial time moments of the original systems. The major disadvantage of such methods is the difficulty in deciding the dominant poles of the original system, which should be retained in the reduced order models. Retaining the poles closest to the imaginary axis need not be always the best choice. Sinha et al, [5] used the clustering technique but the serious limitation of this method is the number and position of zeros of the original system sometimes decides the minimum order of the reduced order model. The major disadvantage of such methods is in deciding the clusters of poles hence cannot generate unique models.
Some methods based on Eigen Spectrum which is the cluster of poles of high order system considered to derive the approximant. Pal et al proposed [6] pole-clustering using Inverse Distance Criterion and time-moment matching. Vishwakarma and R.Prasad [7,8] modified the pole clustering by an iterative method. The difficulty with these methods is in selecting poles for the clusters. Mukherjee [9], suggested a method based on Eigen Spectrum Analysis. The Eigen Spectrum consists of all poles of the high order system. The poles of the reduced model are evenly spaced between the first and last poles. Parmar et al [10] proposed a mixed method using Eigen Spectrum Analysis with Factor division algorithm to determine the numerator of the reduced model with known denominator. Parmar et al [11] proposed another mixed method using Eigen Spectrum Analysis equation and Particle Swarm method to find the reduced model. The methods based on eigen spectrum analysis cannot be applied to high order systems having complex poles. Saraswathi et al [17] proposed a method retaining some of the properties of original system based on eigenspectrum. The method can be applied for systems having both real and complex poles unlike the other existing methods [9-11].

Many methods are available for order reduction of high order continuous systems but very few are extended for discrete systems. The proposed method is extended to discrete systems using Tustin approximation [12] to maintain the static error constants identical in both discrete and continuous transfer functions. In the proposed reduced order method the Time moments and Markov parameters are redefined as function of Residues and poles for strictly proper rational transfer functions having real and complex poles. Poles of the Reduced Order Model (ROM) are selected by considering the highest contribution of each pole in redefined Time Moments (RTMs) and lowest contribution in Redefined Markov Parameters (RMPs).

\section{PROPOSED METHOD}

Let the original high order transfer function of a linear time invariant discrete system of $n^{\text {th }}$ order be

$$
G(z)=\frac{N(z)}{D(z)}=\frac{c_{m} z^{m}+c_{m-1} z^{m-1}+\cdots \ldots+c_{1} z+c_{0}}{z^{n}+d_{n-1} z^{n-1}+d_{n-2} z^{n-2}+\cdots \ldots+d_{1} z+d_{0}} ; m<n
$$

Let the original high order transfer function of a linear time invariant discrete system of $n^{\text {th }}$ order in continuous form using Tustin approximation be 


$$
G(s)=\frac{N(s)}{D(s)}=\frac{b_{m} s^{m}+b_{m-1} s^{m-1}+\cdots \ldots+b_{1} s+b_{0}}{s^{n}+a_{n-1} s^{n-1}+a_{n-2} s^{n-2}+\cdots \ldots+a_{1} s+a_{0}} ; m<n
$$

where $D(s)=\prod_{i=1}^{n}\left(s+\lambda_{i}\right) ; n$ is the number of poles and $N(s)=\prod_{i=1}^{m}\left(s+\delta_{i}\right) ; m$ is the number of zeros, $\lambda_{i}=\left(\alpha_{i} \pm\right.$ $\left.j \beta_{i}\right)$ and $\delta_{i}=\left(\varepsilon_{i} \pm j \gamma_{i}\right)$ of high order system $G(s)$. The poles and zeros may be real and/or complex. If they are complex, they occur in conjugate pairs.

The reduced order model of $k^{\text {th }}$ order using proposed new algorithm in continuous form is defined as

$$
R_{k}(s)=\frac{N_{k}(s)}{D_{k}(s)}=\frac{p_{r} s^{r}+p_{r-1} s^{r-1}+\cdots \ldots+p_{1} s+p_{0}}{s^{k}+q_{k-1} s^{k-1}+q_{k-2} s^{k-2}+\cdots \ldots+q_{1} s+q_{0}} ; k<n \ldots
$$

where $D_{k}(s)=\prod_{i=1}^{k}\left(s+\dot{\lambda}_{i}\right)$; $\mathrm{k}$ is the number of poles and $N_{k}(s)=\prod_{i=1}^{m}\left(s+\delta_{i}\right) ; \mathrm{r} \leq \mathrm{m}, \mathrm{r}$ is the number of zeros, $\dot{\lambda}_{i}=\left(\alpha_{l} \pm \jmath \beta_{l}\right)$ and $\delta_{i}=\left(\varepsilon_{l} \pm J \gamma_{l}\right)$ of reduced order model $R_{k}(s)$. In the reduced model poles and zeros may be real and/or complex. If they are complex, they occur in conjugate pairs as mentioned for original system.

Using Tustin approximation the reduced order linear time invariant discrete model of $k^{\text {th }}$ order is

$$
R_{k}(z)=\frac{N_{k}(z)}{D_{k}(z)}=\frac{u_{r} s^{r}+u_{r-1} s^{r-1}+\cdots \ldots+u_{1} s+u_{0}}{s^{k}+v_{k-1} s^{k-1}+v_{k-2} s^{k-2}+\cdots \ldots+v_{1} s+v_{0}} ; k<n
$$

We know that the power series expansion of $G(s)$ about $s=0$ is

$G(s)=C_{0}+C_{1} s+C_{2} s^{2}+\cdots=\sum_{i=0}^{\infty} C_{i} s^{i}$

Where $C_{i}=\frac{(-1)^{i}}{i !} R T M_{i} ; i=0,1,2,3, \ldots \ldots$

The expansion of $G(s)$ about $s=\infty$ is

$$
\begin{aligned}
G(s) & =\mu_{0} s^{-(n-m)}+\mu_{1} s^{-(n-m+1)}+\mu_{2} s^{-(n-m+2)}+\cdots \\
& =\sum_{i=0}^{\infty} \mu_{i} s^{-(n-m+i)}
\end{aligned}
$$

Where $\mu_{i}=R M P_{i} ; I=0,1,2,3, \ldots$.

i) Considering the original high order system $G(s)$ with distinct poles

Define the expressions for redefined time moments (RTMs) as

$$
R T M_{i}=\sum_{j=1}^{n} x_{i j} ; i=0,1,2, \ldots
$$

where $x_{i j}=i ! \frac{P_{j}}{\lambda_{j}^{(i+1)}} ; i=0,1,2, \ldots$

Define Redefined Markov Parameters (RMPs) as

$$
R M P_{i}=\sum_{j=1}^{n} y_{i j}
$$

where $y_{i j}=P_{j} \lambda_{j}^{i} ; i=0,1,2, \ldots$ and $P_{j}$ are residues.

ii) If $G(s)$ is having ' $r$ ' repeated poles

$G(s)=\frac{b_{m} s^{m}+b_{m-1} s^{m-1}+\cdots \ldots+b_{1} s+b_{0}}{\left(s+\lambda_{1}\right)^{r}\left(s+\lambda_{r+1}\right) \ldots \ldots .\left(s+\lambda_{n}\right)} ; m<n$

Define the expressions for redefined time moments (RTMs) as where $E T_{i x}=\left[\sum_{x=1}^{r-1} \frac{P_{(x+1)}}{\lambda_{1}^{(i+x+1)}}\left\{\prod_{j=1}^{x} \frac{(-1)^{j}(i+j)}{x !}\right\}\right]$ and $\mathrm{P}_{\mathrm{j}}$ are residues.

Define Redefined Markov Parameters (RMPs) as

$R M P_{i}=\left[P_{1} \lambda_{1}^{i}+\left[E M_{i x}\right]+\sum_{k=r+1}^{n} P_{k} \lambda_{k}^{i}\right] ; i=0,1,2, .$.

where $E M_{i x}=\left[\sum_{x=1}^{r-1} P_{(x+1)} \lambda_{1}^{(i-x)}\left\{\prod_{j=1}^{x} \frac{(i-j+1)}{x !}\right\}\right] ; E_{i x}=0$ if $i \leq x$ and $P_{j}$ are residues.

The denominator polynomial $\mathrm{D}_{k}(\mathrm{~s})$ of the $k^{\text {th }}$ order reduced model is obtained by selecting poles with the highest contribution in RTMs and lowest contribution in RMPs according to their contribution weight age as shown in Table I.

Table I : Contributions of individual poles

\begin{tabular}{|l|l|l|l|l|l|l|l|l|}
\hline Parameters & $\lambda_{1}$ & $\lambda_{2}$ & $\lambda_{3}$ & $\ldots$ & $\lambda_{j}$ & $\ldots$ & $\lambda_{n}$ & Sum \\
\hline RTMs & $x_{i 1}$ & $x_{i 2}$ & $x_{i 3}$ & $\ldots$ & $x_{i j}$ & $\ldots$ & $x_{i n}$ & $\mathrm{RTM}_{i}$ \\
\hline RMPs & $y_{i 1}$ & $y_{i 2}$ & $y_{i 3}$ & $\ldots$ & $y_{i j}$ & $\ldots$ & $y_{i n}$ & $\mathrm{RMP}_{i}$ \\
\hline
\end{tabular}

Where $x_{i j}$ is the contribution of pole $\lambda_{j}$ in $R T M_{i}$ and $y_{i j}$ is the contribution of pole $\lambda_{j}$ in $R M P_{i}$. The numerator polynomial, $N_{k}(s)$ of the $k^{\text {th }}$ order reduced model is obtained by retaining the first few initial RTMs and RMPs of the original system as follows:

$$
N_{k}(s)=\sum_{i=0}^{r_{1}} p_{i} s^{i}+\sum_{i=1}^{r_{2}} p_{\left(r_{1+} i\right)} S^{\left(r_{1}+i\right)} ; r=r_{l}+r_{2}, \quad r \leq m
$$

and $r_{l} \geq 1$.

where $p_{i}=\sum_{i=0}^{j} q_{i} C_{(j-i)} ; j=0,1,2,3, \ldots, r_{l} ; r_{1}$ is number of RTMs

and $p_{(r-j)}=\sum_{i=0}^{j} q_{(r-i)} \mu_{(j-i)} ; j=0,1,2,3, \ldots, r_{2} ; r_{2}$ is number of RMPs

$r_{2}$, the number of RMPs is zero if $r_{l}$ is considered. If $n-m<$ 1 , naturally $r_{2}=0$.

\section{EXAMPLE}

Considering a sixth order discrete system described by the transfer function given as

$$
\begin{gathered}
G_{1}(z) \\
0.3277 z^{6}+0.9195 z^{5}+1.038 z^{4}+0.5962 z^{3} \\
+0.1618 z^{2}+0.006986 z-0.005308 \\
=\frac{0.2889 z^{4}-0.08251 z^{3}-0.04444 z^{2}}{z^{6}+1.129 z^{5}+0.0476 z}
\end{gathered}
$$

Using the Tustin transformation $G_{1}(z)$ is transformed to $G_{1}(s)$ with sampling time $\mathrm{T}=1$.

$$
\begin{aligned}
& G_{1}(s) \\
& =\frac{s^{5}+15.6 s^{4}+124.2 s^{3}+510.3 s^{2}+1166 s+959.3}{s^{6}+21 s^{5}+175 s^{4}+735 s^{3}+1624 s^{2}+1764 s+720}
\end{aligned}
$$

The poles of $\mathrm{G}_{1}(\mathrm{~s})$ are $-\lambda_{1}=-1,-\lambda_{2}=-2,-\lambda_{3}=-3,-\lambda_{4}=-$ $4,-\lambda_{5}=-5$ and $-\lambda_{6}=-6$. The contributions of individual poles are derived from equ.(7) for RTMs and from equ.(8) (non zero terms) for RMPs. These contributions are tabulated in Tables II and III. Poles having highest contribution in RTMs and 
lowest contribution in RMPs according to their contribution weight age are $-\lambda_{z_{1}}=-1$ and $-\lambda_{z_{3}}=-4$.

Table II Contribution of poles in RTMs and RMPs of HOS

\begin{tabular}{|c|c|c|c|}
\hline $\begin{array}{ll} & \text { RTMs/RMP } \\
\text { s } & \end{array}$ & $\lambda_{1}$ & $\lambda_{2}$ & $\lambda_{3}$ \\
\hline $24 \mathrm{RTM}_{0}=1.33$ & $\begin{array}{ll}1.616 \\
7\end{array}$ & 2.2396 & -7.7444 \\
\hline${ }_{48} \mathrm{RTM}_{1}=1.64$ & $7 \begin{array}{ll}1.616 \\
7\end{array}$ & 1.1198 & -2.5815 \\
\hline${ }_{00} \mathrm{RMP}_{0}=1.00$ & $\begin{array}{ll}7 & 1.616\end{array}$ & 4.4792 & -23.233 \\
\hline $\mathrm{RMP}_{1}=-5.4$ & $\begin{array}{c}- \\
1.6167\end{array}$ & 8.9583 & 69.700 \\
\hline
\end{tabular}

Table III Contribution of poles in RTMs and RMPs of HOS

\begin{tabular}{|c|l|l|}
\hline$\lambda_{4}$ & $\lambda_{5}$ & $\lambda_{6}$ \\
\hline 10.8146 & -8.4433 & 2.8493 \\
\hline 2.7036 & -1.6887 & 0.4749 \\
\hline 43.2583 & -42.2167 & 17.0958 \\
\hline- & 211.0833 & -102.575 \\
\hline
\end{tabular}

For the second order model with two poles in ESZ, Denominator polynomial is

$$
\begin{aligned}
\mathrm{D}_{2}(s) & =(s+1)(s+4) \\
& =s^{2}+5 s+4
\end{aligned}
$$

Numerator of the ROM is obtained using equation (11) matching first two RTMs of the original system from Table II.

$$
\begin{aligned}
\mathrm{N}_{2}(\mathrm{~s}) & =(1.3324 \text { X 4) }+\{(1.3324 \text { X 5 })+(-1.6448 \text { X 4) }\} \mathrm{s} \\
& =5.329+0.8244 \mathrm{~s}
\end{aligned}
$$

The transfer function of the second order reduced model is

$$
\text { IV. } \quad R_{1-1}(s)=\frac{0.8244 s+5.329}{s^{2}+5 s+4}
$$

The conversion of continuous transfer function to discrete $\mathrm{R}_{1-1}(\mathrm{~s})$ to $\mathrm{R}_{1-1}(\mathrm{z})$ is done using the Tustin transformation with Sampling time 1 . The second order reduced discrete model is

$R_{1-1}(z)=\frac{0.3052 z^{2}+0.5922 z+0.2869}{z^{2}-0.1111}$

The second order model by Farsi et al [13] is

$R_{1-1}^{F}(z)=\frac{0.589 z-0.4495}{z^{2}-1.428 z+0.5329}$

The step response of the proposed second order discrete model $R_{2^{-} l}(z)$ and second order discrete model of Farsi et al [13] $\mathrm{R}_{2-1}^{\mathrm{F}}(z)$ are compared with original discrete system $G_{l}(z)$ in Fig.1. The step response by proposed method is following the original very closely when compared Farsi et al[13].

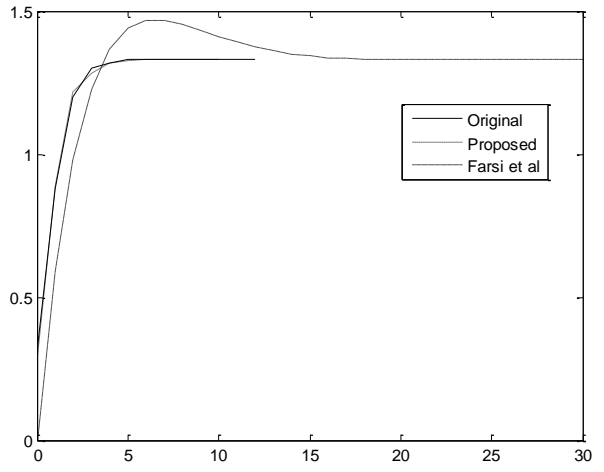

Fig.1 Comparison of step responses of $\mathrm{G}_{1}(\mathrm{z}), \mathrm{R}_{2-1}^{\mathrm{F}}(z)$ and $\mathrm{R}_{2-1}(\mathrm{z})$

\section{CONCLUSIONS}

Conclusions and Future Research: In this paper, an effective procedure to determine the reduced order model of higher order linear time invariant discrete systems is presented. Numerator and denominator polynomials of reduced order model are obtained by redefining the time moments of the original high order system. The stability of the original system is preserved in the reduced order model as the poles are taken from the original system. The method produces a good approximation when compared with other methods. The method is applied for real, complex and repeated poles of continuous [14] and discrete systems and the work is in progress to make it generalize for interval systems.

\section{REFERENCES}

[1] Sinha, N.K. and Pille,W., "A new method for order reduction of dynamic systems", International Journal of Control, Vo1.14, No.1, pp.111-118, 1971.

[2] Sinha, N.K. and Berzani, G. T., "Optimum approximation of high-order systems by low order models", International Journal of Control, Vol.14, No.5, pp.951-959, 1971.

[3] Davison, E. J., "A method for simplifying linear dynamic: systems", IEEE Trans. Automat. Control, vol. AC-11, no. 1, pp.93-101, 1966.

[4] Marshall, S. A., "An approximate method for reducing the order of a linear system", International Journal of Control, vol. 10, pp.642-643, 1966.

[5] Mitra, D., "The reduction of complexity of linear, time-invariant systems", Proc. 4th IFAC, Technical series 67, (Warsaw), pp.19-33, 1969.

[6] J.Pal, A.K.Sinha and N.K.Sinha, "Reduced order modeling using pole clustering and time moments matching", Journal of the Institution of engineers (India), Pt.EL, Vol pp.1-6,1995.

[7] C.B.Vishwakarma, R. Prasad, "Clustering methods for reducing order of linear systems using Padé Approximation", IETE Journal of Research, Vol.54, Issue 5, pp.326-330, 2008.

[8] C.B.Vishwakarma, R. Prasad, "MIMO system reduction using modified pole clustering and Genetic Algorithm", personal correspondence.

[9] S.Mukherjee, "Order reduction of linear systems using eigen spectrum analysis", Journal of electrical engineering IE(I),Vol 77, pp 76-79,1996.

[10]G.Parmar, S.Mukherjee, R.Prasad, "System reduction using factor division algorithm and eigen spectrum analysis", Applied Mathematical Modelling, pp.2542-2552, Science direct, 2007.

[11]G.Pamar, S.Mukherjee, "Reduced order modeling of linear dynamic systems using Particle Swarm optimized eigen spectrum analysis", International journal of Computational and Mathematical Sciences, pp.4552, 2007.

[12]Franklin, G.F.J.D.Powell, and M.L.Workman, "Digital control of Dyanmic systems", second, Addisan-Wesley 1990. 
[13]Farsi M.,Warwick K. and Guilandoust M. "Stable reduced order models for discrete time systems", IEE Proceedings, Vol.133, pt.D, No.3,pp.137141, May 1986.

[14]G.Saraswathi, "An extended method for order reduction of Large Scale Systems", Journal of Computing, Vol.3, Issue.4, April, 2011.

[15]G. Saraswathi, K.A. Gopala Rao and J. Amarnath, "A Mixed method for order reduction of interval systems having complex eigenvalues", International Journal of Engineering and Technology, Vol.2, No.4, pp.201-206, 2008.

[16]G.Saraswathi, "Some aspects of order reduction in large scale and uncertain systems", Ph.D. Thesis. 